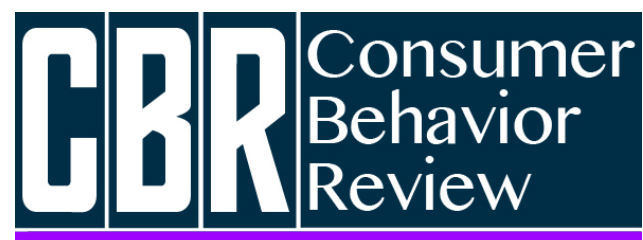

Revista Comportamento do Consumidor
Brito, M. G., Spanhol-Finocchio, C. P., Brun, H. M., \& Mendonça, M. T. (2020). Imagem e Satisfação dos Consumidores do Mercado Municipal de Campo GrandeMS. Consumer Behavior Review, 4(2), 176-188.
ISSN: 2526-7884

Editor: Prof. Dr. Marconi Freitas da Costa Journal's e-mail: cbr@ufpe.br
Evaluation: Double blind review

Received: 20 de janeiro de 2020

Accepted: 28 de abril de 2020

\title{
IMAGEM E SATISFAÇÃO DOS CONSUMIDORES DO MERCADO MUNICIPAL DE CAMPO GRANDE-MS
}

\author{
Image and consumer satisfaction in the Municipal Market, Campo Grande, Mato Grosso do Sul \\ state, Brazil
}

\author{
Mauricéia Gomes de Brito ${ }^{1}$ \\ ORCID: https://orcid.org/0000-0002-0168-8025 \\ E-mail: mauridebrito@hotmail.com \\ Caroline Pauletto Spanhol-Finocchio ${ }^{2}$ \\ ORCID: https://orcid.org/0000-0002-0979-4594 \\ E-mail: caroline.spanhol@ufms.br \\ Handerson Molin Brun² \\ ORCID: https://orcid.org/0000-0001-5699-0697 \\ E-mail: handmolin@hotmail.com \\ Marilza Trindade Mendonça² \\ ORCID: https://orcid.org/0000-0002-1475-7683 \\ E-mail: marilzamendonca@gmail.com
}

\begin{abstract}
${ }^{1}$ Departamento de Administração, Universidade Federal de Mato Grosso do Sul, Campo Grande, Brasil
${ }^{2}$ Programa de Pós-Graduação em Administração, Universidade Federal de Mato Grosso do Sul, Campo Grande, Brasil
\end{abstract}

\section{Resumo}

Conhecer os atributos de imagem de loja mais valorizados pelo consumidor é necessário para orientar investimentos e fortalecer a lembrança da loja na mente do consumidor. 0 objetivo do estudo foi avaliar a imagem de loja

\begin{abstract}
Knowing the store image attributes most valued by the consumer is necessary to guide investments and strengthen the store's memory in the consumer's mind. The objective of the study was to evaluate the
\end{abstract}


e a satisfação do consumidor do Mercado Municipal de Campo Grande-MS. A coleta de dados foi realizada online com uma amostra de 262 consumidores. Os atributos que apresentaram as melhores avaliações, na percepção dos consumidores, foram: mercadoria, layout e atendimento, indicando que o local possui uma grande variedade de mercadorias, diferencial no atendimento e um boa organização e exposição dos produtos.

Palavras-chave: Satisfação, Comportamento, Imagem de loja, Mercado Municipal, Mercadão. store image and consumer satisfaction of the Municipal Market of Campo Grande-MS. Data collection was carried out online with a sample of 262 consumers. The attributes that presented the best evaluations, in the perception of consumers, were: merchandise, layout and service, indicating that the place has a wide variety of goods, differential in service and a good organization and exhibition of products.

Keywords: Satisfaction, Behavior, Image story, Municipal market, Mercadão.

Esta obra está licenciada com uma Licença Creative Commons Atribuição 4.0 Internacional.

\section{INTRODUÇÃO}

O varejo é um importante setor da economia global. Segundo dados da Associação Brasileira de Supermercados (ABRAS), o setor varejista brasileiro de alimentos respondeu por aproximadamente $5,2 \%$ do Produto Interno Bruto brasileiro em 2018, totalizando receita bruta de aproximadamente R $\$$ 356 bilhões no mesmo ano (ABRAS, 2019).

Os supermercados, as feiras e os mercados públicos são importantes canais de distribuição de alimentos, sendo necessário compreender o consumidor e os motivos que o levam a escolher entre um ou outro formato de loja. O comportamento do consumidor caracteriza-se por um conjunto de atividades físicas e psicológicas que ocorrem no momento da seleção e compra de um determinado bem ou serviço, sendo que essas atividades se iniciam antes do momento da decisão de compra e vai além do ato de comprar (Watanabe, 2014). Trata-se de um processo abrangente que envolve fatores sociais, pessoais e ambientais. Entre os fatores ambientais, que podem exercer influência no comportamento de compra dos consumidores está a imagem de loja.

A imagem de loja diz respeito ao que está gravado na mente dos consumidores com relação a loja. Foi definida, primeiramente, por Martineau (1958) como a forma como a loja é lembrada pelo consumidor. Assim, o conhecimento dos atributos que compõe a imagem de loja torna-se necessário para fortalecer a lembrança, de maneira positiva, na mente do consumidor, bem como orientar investimentos nessa área.

Diversas pesquisas sobre imagem de loja foram desenvolvidas, considerando como objeto de estudo empresas de diferentes setores, tais como restaurantes, supermercados e lojas de departamentos. Este estudo, por outro lado, buscou avaliar a imagem de loja e a satisfação do consumidor do Mercado Municipal de Campo Grande-MS, ponto turístico do município e que será tombado como patrimônio histórico e cultural.

No Mercado Municipal Antônio Valente, mais conhecido como Mercadão, situado na cidade de Campo Grande-MS são comercializados produtos alimentícios, utensílios para o dia a dia e artesanatos, constituindo-se um importante canal de distribuição de gêneros alimentícios. Possui 144 bancas e 79 boxes. Foi inaugurado em 1958, e possui um fluxo de até 5 mil pessoas por dia. Segundo Queiroz (2019), o Mercadão é berço da cultura, culinária e ponto turístico de Campo Grande-MS.

Este estudo está estruturado em cinco seções, sendo a primeira esta introdução. Na segunda encontra-se o referencial teórico, enquanto a terceira é dedicada aos procedimentos metodológicos. Na quarta seção os resultados são apresentados e discutidos. Na quinta seção são apresentadas as considerações finais. 


\section{REFERENCIAL TEÓRICO}

Neste tópico serão abordados os conceitos que fundamentam esta pesquisa: conceito de imagem de loja, os atributos de imagem de loja, a relação direta entre imagem de loja e a satisfação do consumidor e a história do Mercado Municipal de Campo Grande-MS.

\section{Imagem de loja}

Segundo Parente (2007), a identidade de uma loja está ligada à sua imagem, sendo que o conhecimento e a aplicação de seus conceitos podem gerar resultados positivos e aumento de vendas. Por outro lado, uma interpretação imprecisa sobre layout, exposição de produtos, ambiente interno e externo pode afetar seus resultados.

Martineau (1958) apud Graciola (2015) foi o primeiro autor a escrever sobre imagem de loja em seu livro The Personality of the Retail Store. 0 autor afirma que a loja possui uma personalidade capaz de influenciar os seus consumidores criando uma empatia entre eles através de diversos atributos físicos e psicológicos, definindo um conceito em suas mentes, contribuindo assim para a escolha de determinada loja. Para Bloemer e Ruyter (1998), a imagem de loja é um conjunto de ações pautado nas observações de diversos atributos significativos.

Segundo Lindquist (1974-75), a imagem de loja é um conjunto de dimensões que formam a estrutura de uma determinada atividade. Além disso, o autor afirma que a imagem de loja não é estática, mas se altera, pois, essas dimensões se modificam ao longo do tempo. Essas dimensões podem estar ligadas a novas experiências, situação econômica ou social (Theodoridis \& Chatzipanagiotou, 2009).

Alguns autores definem que a imagem de loja é uma construção complexa, composta por atributos funcionais, atitudes e percepções dos consumidores (Stern, Zinklan \& Jaju, 2001). Sob a ótica do consumidor, a imagem de loja é o total de atributos que vem à mente sobre determinada loja, baseado nas características físicas da loja e de um mix de produtos e atributos psicológicos (AMA, 2014). Para os autores Silva e Giraldi (2012), a imagem da loja é a soma das percepções que o consumidor possui de uma loja, ou seja, é a soma do que eles conhecem sobre os atributos da loja devido a experiência com a mesma.

Conforme mencionado, um dos estudos seminais sobre o tema foi elaborado por Martineau (1958) apud Graciola (2015). A pesquisa do referido autor teve como objetivo verificar quais motivos levavam os consumidores a optarem por uma loja em detrimento a outra. Martineau e listou os atributos funcionais da loja que incluem a localização, faixas de preço e seleção de mercadorias e ilustra os atributos psicológicos que contribuem para a criação da imagem da loja: layout e arquitetura da loja, símbolos (emblemas) e cores, publicidade e pessoal de vendas da loja.

0 estudo de Theodoridis e Chatzipanagiotou (2009), realizado na Grécia, verificou o efeito dos atributos de imagem de loja na satisfação do consumidor testando seis atributos: produtos, preços, atmosfera, pessoal, merchandising e conveniência. Os atributos mais relevantes foram: produto, preço, pessoal e conveniência. No Brasil, o estudo de Watanabe, Lima-Filho e Torres (2013) utilizou os mesmos atributos: produto, preço, atmosfera, pessoal, merchandising e conveniência para analisar a imagem de loja. Nesse estudo, os atributos significativos foram: preço, produtos e pessoal, sendo o preço o de maior peso. A relação entre imagem e satisfação foi confirmada nestes estudos.

Ainda com relação aos atributos de imagem de loja, a pesquisa de Rojo (1998) teve como objetivo identificar os atributos mais relevantes apontados pelos consumidores na avaliação de supermercados e o resultado foi que o preço, ofertas e promoções, atendimento, qualidade, variedade, crédito, frente de caixa e perecíveis foram os mais relevantes. O estudo de Lima-Filho (1999) identificou que os atributos mais valorizados pelos consumidores de supermercados são: substituição de produtos vencidos ou estragados e a limpeza da loja. 0 estudo apontou também que a exposição e organização dos produtos e a qualidade dos produtos hortifrutículas são significativos para a percepção de valor do consumidor. Campolina et al. (2007) identificou que os atributos que mais influenciam o processo de compra/escolha são: preço, opções de pagamento, variedade e qualidade dos produtos, tamanho da loja, higiene, atendimento, localização estacionamento, premiações e promoções. 
A partir dos estudos revisados, observou-se que vários estudos apresentam atributos diferentes para analisar a imagem da loja. No estudo da Graciola (2015), a autora realizou um levantamento bibliográfico sobre os atributos mais utilizados em estudos com foco no varejo, tendo como resultado: atmosfera da loja (88\%), nível de preço (59\%), atendimento (59\%) e mercadoria "variedade de produtos" (53\%). A atmosfera da loja devido a relevância que teve no estudo foi desmembrada em subitens para melhor compreensão, sendo eles: layout, ambiente, percepção de densidade e projeto arquitetônico.

\section{Construtos de imagem de loja}

\section{Atmosfera de loja}

Segundo Parente (2007), a atmosfera de loja é desenvolvida utilizando recursos de apresentação, tanto interna como externa da loja, opções de layout e exposição dos produtos. A atmosfera de loja está ligada a imagem que o consumidor tem da loja, motivando a sua permanência no local por mais tempo e a explorar outros departamentos.

Segundo Van Der Vyver (2008), os gestores podem manipular os efeitos do ambiente a fim de influenciar o comportamento do consumidor através da atmosfera de loja, fazendo com que seu subconsciente reconheça a atmosfera da loja conforme a frequência que visita o local tornando-o familiar (Theodoridis \& Chatzipanagiotou, 2009). Para este estudo optou-se por abordar os componentes de atmosfera de loja propostos por Graciola (2015): ambiente, percepção de densidade, layout e o projeto arquitetônico; abordados a seguir.

\section{Ambiente}

0 ambiente é composto por vários fatores, entre eles cheiro, iluminação, temperatura e música que apresentam efeitos nas decisões de compra (Baker, Berry \& Parasuraman, 1988; Baker, Levy \& Grewal, 1992). Segundo Soars (2009), os sentidos geram forte efeito no consumidor através do olfato, audição, paladar e toque conforme a percepção do ambiente. Assim, a permanência do consumidor no ambiente pode ser influenciada pela combinação de cores e iluminação (Soars, 2009). 0 autor afirma que é necessário um equilíbrio na música utilizada, na qualidade e a altura do som, pois a não observância desses fatores pode criar impactos negativos, contribuindo até para a saída do consumidor de determinado ambiente.

\section{Layout}

Um bom layout aumenta a permanência dos consumidores e influencia as compras por impulso ou não programadas (Mohan, Sivakumaran \& Shama, 2013). Além de estar positivamente relacionado com a intenção de compra (Grewal \& Baker, 1994). A otimização do espaço depende da criação de corredores capazes de conduzir o cliente por produtos expostos de forma atraente, proporcionando conforto aos consumidores, assegurando um fluxo eficiente e proporcionando um incremento nas vendas, consequentemente aumentando os lucros (Batista \& Escobar 2020; Morgan, 2011; Silva et al., 2020).

\section{Percepção de densidade}

Um dos fatores que vem sendo estudado é o efeito crowding, conceito multidimensional que é composto pela percepção de densidade humana e espacial em um determinado ambiente (Hui \& Bateson, 1991). Destaca-se que existe a tendência dos consumidores sentirem-se constrangidos e confinados no ambiente quando há percepção de sobrecarga pela alta densidade (Wang, Chang \& Ysong, 2012). 0 autor afirma ainda que essa percepção pode influenciar negativamente na satisfação do consumidor.

\section{Projeto arquitetônico}

Projetos arquitetônicos são edificações que são vistas como objetos de marketing (Glendinning, 2004). A aparência de uma nova loja é vista como um ponto de referência para a população (Jencks, 2005) e se tornam ícones na edificação existente nessa área (Julier, 2005). Um bom 
projeto arquitetônico comunica a imagem da marca e revela a identidade da loja, tanto externa como internamente, sendo percebida pelo consumidor no momento da compra (Din, 2000). A aparência das fachadas contribue para chamar a atenção do consumidor e despertar o interesse em entrar na loja, criando suas primeiras impressões sobre ela.

A coerência entre pinturas e texturas das paredes com o ambiente e a exposição dos produtos contribuem para a permanência do consumidor no local, causando bem-estar. A limpeza do local causa sensação de conforto no ambiente (Baker, Levy \& Grewal, 1992).

\section{Variedade de produtos / Mercadorias}

Uma variedade de produtos causa uma maior percepção de qualidade e da imagem da loja no contexto do varejo alimentar (Lindquist, 1974-75). Segundo Mittal e Mittal (2008), essa variedade implica na escolha de determinada loja, contribuindo para que os consumidores comprem mais, caso tenham planejado antecipadamente suas compras, criando uma vantagem para o lojista. Entretanto, os consumidores possuem preferências individuais que precisam ser supridas a partir do entendimento do lojista dessas necessidades (Morrison, 2006).

Baltas e Papastathopoulou (2003), em seu estudo aplicado em um supermercado na Grécia, apontou que a variedade de produtos influencia na decisão de compra. Entretanto, manter um equilíbrio na variedade é essencial para não causar sobrecarga para o consumidor, para não influenciar de forma negativa trazendo sentimento de frustração (Iyengar \& Lepper, 2000).

A exposição e a organização dos produtos nas prateleiras devem levar em conta a acessibilidade aos consumidores, pois se não estiverem, podem acarretar em custos para o lojista e diminuição do valor percebido (Mittal \& Mittal, 2008).

\section{Nível de Preço}

A percepção do nível de preço é um dos fatores que determinam a imagem que o consumidor possui da loja (Lindquist, 1974-75). A disposição dos consumidores em pagar um preço mais alto em um produto diferenciado está associada a um produto de boa imagem em que percebem maior qualidade e status (Swani \& Yoo, 2010). Por outro lado, o autor afirma, ainda, que os consumidores tendem a buscar produtos ou marcas com preços mais acessíveis onde for oferecido o melhor preço.

\section{Atendimento}

0 atendimento ao cliente é um dos elementos mais importantes no varejo, pois causam um impacto positivo na imagem de loja $(\mathrm{Hu}, 2011)$ sendo o mesmo de vital importância para proporcionar ao consumidor uma experiência memorável (Aguiar \& Policarpo, 2018). Essa associação é positiva pelo fato dos consumidores sentirem-se em um ambiente amigável, receberem um atendimento cortês e educado, gerando a satisfação do cliente (Jinfeng \& Zhilong, 2009).

Com os diferentes formatos de compras, em que não há interação com os funcionários, investir em um bom atendimento quando há interação se torna um diferencial para o lojista (Harris, Baron \& Parker, 2000). A aparência dos colaboradores também contribui na percepção do consumidor como uniformes, boa aparência e simpatia (Kim \& Kim, 2012). A oportunidade de criar um bom relacionamento dos funcionários com os clientes contribui para uma boa imagem de loja na percepção dos seus consumidores (Hu \& Jasper, 2006).

\section{A relação entre imagem de loja e a satisfação}

A satisfação dos clientes vem sendo definida como a avaliação de uma série de resultados como desempenho de vendas, retenção e fidelização de clientes Gomez, McLaughlin e Wittink (2004), sendo também uma avaliação pós-compra (Oliver, 1980,1981; Costa \& Falcão, 2018). Assim a satisfação do cliente é definida como "a avaliação global do cliente da experiência na loja" MacIntosh e Lockshin (1997, p. 489), apud Theodoris e Chatzipanagioutou (2009).

Theodoris e Chatzipanagioutou (2009), em seu estudo em um supermercado grego, confirmou a relação direta entre quatro atributos de imagem de loja que impactaram significativamente na satisfação de seus clientes: pessoal, preço, produto e conveniência no interior da loja. A satisfação do 
cliente é de suma importância, pois é responsável em irrigar monetariamente todo o processo de comercialização do setor, sem o consumidor o não há justificativa para a comercialização do produto (Brun, Rodrigues, Finocchio, \& Souza, 2019).

\section{O Mercado Municipal de Campo Grande-MS}

O Mercado Municipal Antônio Valente, mais conhecido como Mercadão, nasceu de uma feira livre e foi inaugurado em 30 de agosto de 1958. É considerado um ponto turístico e cultural na cidade, possui uma área construída de cerca de $2.494 \mathrm{~m}^{2}$, área essa que foi doada pelo então senhor Antônio Valente, funcionário da Noroeste do Brasil, estrada de ferro.

O Mercadão é composto por 144 bancas e 79 boxes que reúne cerca de 600 trabalhadores e desde 1983 é administrada pela Associação dos Comerciantes do Mercado Municipal de Campo Grande - MS (ASSOCIMEC). O Mercadão já passou por três reformas sendo a última em 2015, com a ampliação do estacionamento.

Considerado como um polo comercial e varejista é responsável por manter a tradição campograndense. Em seu espaço encontram-se diversos produtos, entre eles ingredientes típicos da região, erva para tereré, que é amplamente consumida na região. Também é possível encontrar carnes bovinas, suínas e peixes, frutas, verduras, sacolão e doces, ervas medicinais, condimentos e lanchonetes. O Mercadão é referência na cidade para passeios e compras, sendo muito indicado para os turistas pelos moradores da cidade, um ponto de encontro aos fins de semana para saborear os pastéis e a música regional.

\section{MÉTODO}

A pesquisa desenvolvida é classificada como quantitativa-descritiva (Malhotra, Birks \& Wills, 2012), sendo aquela que apresenta maior potencial para realizar descrições sobre o mercado (Tonetto, Brust-Renck \& Stein, 2014). Optou-se por utilizar a escala Likert de sete pontos, tendo em seus extremos "1. Discordo totalmente" a "7 Concordo totalmente" para avaliar os atributos de imagem da loja e a satisfação dos consumidores do Mercadão Municipal de Campo Grande-MS.

\section{População-alvo e amostragem}

A população-alvo desta pesquisa é composta por consumidores, homens e mulheres, frequentadores do Mercado Municipal de Campo Grande-MS com idade mínima de 18 anos. A amostragem foi não probabilística por conveniência (Malhotra, Birks \& Wills, 2012). Destaca-se que para se ter uma boa análise de dados é necessária uma amostra apropriada. Hair et. al. (2010) recomendam um tamanho mínimo de amostra de cinco vezes o número de itens a serem avaliados. Como o questionário possui 37 itens, a amostra necessária seria de 185 indivíduos. No entanto, foram obtidos 262 questionários, sendo esse quantitativo superior ao mínimo recomendável.

\section{Operacionalização do instrumento de coleta de dados}

0 questionário estruturado utilizado nesta pesquisa (Quadro 1) foi baseado no questionário utilizado por Graciola (2015). A coleta de dados foi realizada por meio de um questionário online, compartilhado por meio de um link em redes sociais, Wattsapp e e-mails. Aos respondentes foi solicitado que compartilhassem e indicassem outros consumidores para participar da pesquisa.

\begin{tabular}{|l|c|c|l|l|}
\hline \multicolumn{3}{|c|}{ Variáveis } & \multicolumn{2}{|c|}{ Itens } \\
\hline \multirow{4}{*}{$\begin{array}{l}\text { Imagem } \\
\text { de loja }\end{array}$} & Layout & LY1 & $\begin{array}{l}\text { O layout do estabelecimento torna fácil chegar às } \\
\text { prateleiras/setores de produtos que eu procuro. }\end{array}$ \\
\cline { 2 - 5 } & $\begin{array}{c}\text { Percepção de } \\
\text { Densidade }\end{array}$ & LY2 & $\begin{array}{l}\text { No geral, o layout torna fácil meu deslocamento no } \\
\text { estabelecimento. }\end{array}$ \\
\cline { 2 - 5 } & \begin{tabular}{l} 
PD2 \\
\cline { 3 - 5 }
\end{tabular} & $\begin{array}{l}\text { Este estabelecimento tem um espaço físico amplo. } \\
\text { facilidade. }\end{array}$ \\
\hline
\end{tabular}




\begin{tabular}{|c|c|c|}
\hline & PD3 & Este estabelecimento é espaçoso. \\
\hline & PD4 & $\begin{array}{l}\text { Este estabelecimento não é enclausurado (fechado } \\
\text { demais). }\end{array}$ \\
\hline \multirow{3}{*}{ Ambiente } & $\mathrm{AB} 1$ & 0 aroma (cheiro) dentro do estabelecimento é agradável. \\
\hline & $\mathrm{AB} 2$ & A iluminação é apropriada. \\
\hline & AB3 & A temperatura ambiente é agradável. \\
\hline \multirow{4}{*}{$\begin{array}{l}\text { Projeto } \\
\text { Arquitetônico }\end{array}$} & PA1 & A arquitetura do estabelecimento tem caráter atraente. \\
\hline & PA2 & A fachada do estabelecimento é atraente. \\
\hline & PA3 & A cor do piso interno do estabelecimento é agradável. \\
\hline & PA4 & O estacionamento possui número de vagas adequado. \\
\hline \multirow{4}{*}{ Atendimento } & AT1 & $\begin{array}{l}\text { Os vendedores possuem boa apresentação ou boa } \\
\text { aparência. }\end{array}$ \\
\hline & AT2 & Os vendedores se preocupam com o consumidor. \\
\hline & AT3 & Os vendedores são amigáveis. \\
\hline & AT4 & Os vendedores são bem informados. \\
\hline \multirow{5}{*}{ Nível de Preço } & NP1 & $\begin{array}{l}\text { Os preços neste estabelecimento geralmente são muito } \\
\text { baixos. }\end{array}$ \\
\hline & NP2 & $\begin{array}{l}\text { Os preços neste estabelecimento geralmente são muito } \\
\text { altos. }\end{array}$ \\
\hline & NP3 & $\begin{array}{l}\begin{array}{l}\text { Você pode comprar produtos baratos neste } \\
\text { estabelecimento. }\end{array} \\
\end{array}$ \\
\hline & NP4 & $\begin{array}{l}\text { Os preços são mais baixos neste estabelecimento em } \\
\text { comparação a outros estabelecimentos. }\end{array}$ \\
\hline & NP5 & $\begin{array}{l}\text { Neste estabelecimento o preço é mais alto em comparação } \\
\text { a outros locais. }\end{array}$ \\
\hline \multirow{5}{*}{ Mercadoria } & ME1 & Este estabelecimento oferece produtos confiáveis. \\
\hline & ME2 & $\begin{array}{l}\text { Este estabelecimento oferece grande variedade de } \\
\text { mercadorias. }\end{array}$ \\
\hline & ME3 & $\begin{array}{l}\text { Este estabelecimento apresenta as prateleiras totalmente } \\
\text { abastecidas. }\end{array}$ \\
\hline & ME4 & $\begin{array}{l}\begin{array}{l}\text { Este estabelecimento oferece mercadorias de alta } \\
\text { qualidade. }\end{array} \\
\end{array}$ \\
\hline & ME5 & Este estabelecimento oferece mercadorias diferenciadas. \\
\hline
\end{tabular}

Fonte: Adaptado de Graciola (2015).

Quadro 1. Variáveis investigadas na pesquisa

Para verificar a satisfação dos consumidores, foi utilizada a seguinte escala (Quadro 2):

\begin{tabular}{|l|c|l|}
\hline \multicolumn{2}{|c|}{ Variáveis } & \multicolumn{1}{c|}{ Itens } \\
\hline \multirow{3}{*}{ Satisfação } & S1 & $\begin{array}{l}\text { Fiquei satisfeito (a) em comprar no Mercado } \\
\text { Municipal. }\end{array}$ \\
\cline { 2 - 3 } & S2 & Foi uma escolha sábia comprar no Mercado Municipal. \\
\cline { 2 - 3 } & S3 & Me proporcionou uma boa experiência. \\
\hline
\end{tabular}

Fonte: Elaborado pelos autores.

Quadro2. Escala para mensuração da satisfação

Os dados foram tabulados e analisados com o auxílio do Microsoft Office Excel. Na próxima seção os dados são apresentados e discutidos.

\section{ANÁLISE E DISCUSSÃO DOS RESULTADOS}

A elaboração desta etapa consistiu na apresentação dos dados e sua análise através da estatística descritiva. Para análise dos dados, foram feitos os cálculos das médias, variância e desvio padrão dos atributos individualmente. 


\section{Caracterização da amostra}

Com o objetivo de identificar o perfil da amostra, foram pesquisadas as seguintes variáveis: faixa etária, estado civil, escolaridade, renda familiar bruta e o número de pessoas que residem na mesma habitação. Verifica-se que a maioria dos respondentes 157 (59,9\%) é do gênero feminino e 105 $(40,1 \%)$ são do gênero masculino (Tabela 1$)$.

\section{Tabela 1}

Gênero dos respondentes

\begin{tabular}{lcc}
\hline Gênero & Frequência & $\%$ \\
\hline Masculino & 105 & 40,1 \\
Feminino & 157 & 59,9 \\
\hline Total da amostra & 262 & 100 \\
\hline
\end{tabular}

Fonte: Dados provenientes da coleta de dados.

A faixa etária predominante está entre 26 e 35 anos com 31,7\% dos respondentes, seguida por $27,9 \%$ respondentes entre 36 a 45 anos, $23,7 \%$ respondentes entre 18 a 25 anos, $11,8 \%$ respondentes entre 45 a 55 anos, 3,8\% respondentes entre 56 a 65 anos e 1,1\% respondentes com 56 anos ou mais, nota-se então que aproximadamente $60 \%$ dos clientes do Mercadão estão na faixa etária dos 26 a 45 anos (Tabela 2).

Tabela 2

Faixa Etária dos respondentes

\begin{tabular}{lcc}
\hline Idade & Frequência & $\%$ \\
\hline $18-25$ & 62 & 23,7 \\
$26-35$ & 83 & 31,7 \\
$36-45$ & 73 & 27,9 \\
$46-55$ & 31 & 11,8 \\
$56-65$ & 10 & 3,8 \\
66 ou mais & 3 & 1,1 \\
\hline Total da amostra & 262 & 100 \\
\hline
\end{tabular}

Fonte: Dados provenientes da coleta de dados.

Referente ao estado civil verificou-se a predominância dos respondentes que se apresentaram como solteiros 39,3\%, 34,7\% declararam-se casados, 14,1\% dos respondentes declararam-se casados com filhos, 6,1\% dos respondentes declararam-se em união estável e 0,4\% respondente declarou-se viúvo (a).

Com relação à escolaridade constatou-se que $32,1 \%$ dos respondentes possuem ensino superior incompleto, $19,1 \%$ possuem ensino superior completo, $16,4 \%$ possuem ensino médio completo, $21,8 \%$ dos respondentes possuem pós-graduação completa, $6,9 \%$ possuem pós-graduação incompleta, $1,5 \%$ possuem ensino médio incompleto, $1,1 \%$ possuem ensino fundamental completo e o mesmo percentual de respondentes possuem ensino fundamental incompleto.

A renda familiar bruta mensal concentrou-se de $\mathrm{R} \$$ : 3941,00 a 7880,00 com 28,6\%, 20,6\% declararam a renda entre $\mathrm{R} \$: 2365,00$ e 3940,00 , a faixa de renda entre $\mathrm{R} \$: 1577,00$ e $\mathrm{R} \$: 2364,00$ com $19,5 \%$ dos respondentes, $9,5 \%$ com renda até $\mathrm{R} \$: 1576,00,12,2 \%$ com renda entre $\mathrm{R} \$: 7881,00$ e $\mathrm{R} \$$ : $12000,00,5,0 \%$ entre $\mathrm{R} \$: 12001,00$ e R\$: 15760,00 e 4,6\% dos respondentes com renda acima de $\mathrm{R} \$:$ 15760,00 .

Em relação ao número de pessoas que moram na mesma residência, predomina-se o número de respondentes que habitam com três pessoas na mesma residência incluindo ele mesmo 31,3\%, em seguida os respondentes que moram com quatro pessoas $26,35 \%, 25,2 \%$ habitam com duas pessoas, $7,6 \%$ dos respondentes moram sozinhos, 5,3\% habitam com 5 pessoas, e 4,2\% habitam com 6 pessoas ou mais.

Referente à frequência que os respondentes costumam comprar no Mercado Municipal o maior número de respondentes 64,1\% raramente compram no Mercado municipal, entretanto conhecem o 
local, 26,0\% dos respondentes compram com menos frequência, 8,0\% compram frequentemente e $1,9 \%$ compram muitas vezes no Mercado Municipal.

\section{Resultados dos atributos de imagem de loja}

Ao analisar a Tabela 3, verifica-se que o atributo mercadoria recebeu as melhores avaliações, indicando que os clientes do Mercado Municipal avaliam positivamente o atributo mercadoria, ou seja, oferecer mercadorias confiáveis, de alta qualidade e diferenciadas, assim como prateleiras abastecidas e uma variedade de mercadorias, são pontos positivos e que devem ser mantidos.

Tabela 3

Média variância e desvio padrão

\begin{tabular}{|c|c|c|c|c|c|c|}
\hline Indicadores & Variáveis & Mín. & Máx. & Média & Variância & Desvio Padrão \\
\hline \multirow{3}{*}{ Layout } & LY1 & 1 & 7 & 4,54580 & 3,12036 & 1,76487 \\
\hline & LY2 & 1 & 7 & 4,59542 & 3,29589 & 1,81428 \\
\hline & PD1 & 1 & 7 & 4,27481 & 3,79315 & 1,94977 \\
\hline Percepção de & PD2 & 1 & 7 & 4,06870 & 3,23154 & 1,79907 \\
\hline \multirow[t]{3}{*}{ Densidade } & PD3 & 1 & 7 & 3,95420 & 3,41392 & 1,85018 \\
\hline & PD4 & 1 & 7 & 4,15267 & 3,72170 & 1,93168 \\
\hline & $\mathrm{AB} 1$ & 1 & 7 & 4,35878 & 3,25769 & 1,80493 \\
\hline \multirow[t]{3}{*}{ Ambiente } & AB2 & 1 & 7 & 4,35878 & 3,26525 & 1,80705 \\
\hline & AB3 & 1 & 7 & 4,28244 & 3,05513 & 1,74758 \\
\hline & PA1 & 1 & 7 & 3,54198 & 3,54284 & 1,88667 \\
\hline Projeto & PA2 & 1 & 7 & 3,17557 & 3,26910 & 1,81303 \\
\hline \multirow[t]{3}{*}{ Arquitetônico } & PA3 & 1 & 7 & 3,29771 & 2,90985 & 1,70954 \\
\hline & PA4 & 1 & 7 & 4,16412 & 3,60852 & 1,90175 \\
\hline & AT1 & 1 & 7 & 3,95802 & 3,12286 & 1,76876 \\
\hline \multirow{4}{*}{ Atendimento } & AT2 & 1 & 7 & 4,39695 & 3,04082 & 1,74277 \\
\hline & AT3 & 1 & 7 & 4,87023 & 2,64679 & 1,62073 \\
\hline & AT4 & 1 & 7 & 4,63740 & 2,67512 & 1,63130 \\
\hline & NP1 & 1 & 7 & 3,25954 & 2,67289 & 1,63809 \\
\hline \multirow[t]{6}{*}{ Nível de Preço } & NP2 & 1 & 7 & 4,53817 & 2,77323 & 1,66220 \\
\hline & NP3 & 1 & 7 & 4,38550 & 2,97008 & 1,72214 \\
\hline & NP4 & 1 & 7 & 3,67557 & 2,93252 & 1,71474 \\
\hline & NP5 & 1 & 7 & 4,29389 & 2,88625 & 1,69784 \\
\hline & ME1 & 1 & 7 & 5,12214 & 1,83402 & 1,33920 \\
\hline & ME2 & 1 & 7 & 5,50000 & 2,04614 & 1,41354 \\
\hline \multirow[t]{3}{*}{ Mercadoria } & ME3 & 1 & 7 & 5,55725 & 1,87933 & 1,35137 \\
\hline & ME4 & 1 & 7 & 4,98473 & 1,85216 & 1,34754 \\
\hline & ME5 & 1 & 7 & 5,21374 & 2,31820 & 1,51117 \\
\hline
\end{tabular}

Fonte: Dados provenientes da coleta de dados.

Em segundo lugar está o atributo layout apontando que os clientes do Mercadão levam em consideração a boa organização e disposição dos produtos em seu espaço físico, esses resultados vão ao encontro dos resultados obtidos por Watanabe, Lima-Filho e Torres (2013) e Graciola (2015). Como destacado pelos autores (Hu, 2011; Jinfeng \& Zhilong, 2009; Theodoridis \& Chatzipanagiotou, 2009; Watanabe, Lima-Filho e Torres, 2013; Rojo, 1998; Graciola, 2015) o atendimento apesar de aparecer em terceiro lugar, é de suma importância para proporcionar uma imagem positiva. Nesse quesito, pode-se concluir que o Mercadão conta com profissionais atenciosos e que possuem conhecimento suficiente para promover uma boa imagem aos seus clientes.

Esta pesquisa revela, também, que o consumidor avalia o preço dos produtos comercializados altos. A percepção de preço alto por parte dos respondentes pode estar associada à sua menor frequência de compra no Mercadão. Entre os atributos que se demonstram frágeis na transmissão de uma boa imagem ao consumidor, podemos destacar o Projeto Arquitetônico, cujo as avaliações se apresentaram abaixo da média, o que sugere que alterações devem ser executadas. 
Os demais atributos de imagem se encontram na média, o que não é considerado um ponto positivo, mas sim um alerta para que sejam observados pela administração do empreendimento, com o intuito de fortalecer a imagem positiva do Mercadão na mente dos consumidores, bem como a intenção de retornar ao estabelecimento. A satisfação dos consumidores do Mercadão revela-se positiva conforme mostra a Tabela 4:

Tabela 4

Média, variância e desvio padrão da variável satisfação

\begin{tabular}{lcccccc}
\hline Indicadores & Variáveis & Mín. & Máx. & Média & Variância & $\begin{array}{c}\text { Desvio } \\
\text { Padrão }\end{array}$ \\
\hline \multirow{2}{*}{ Satisfação } & S1 & 1 & 7 & $\mathbf{5 , 3 1 2 9 8}$ & 2,08878 & 1,43083 \\
& S2 & 1 & 7 & 5,06489 & 2,26086 & 1,49315 \\
& S3 & 1 & 7 & 5,12595 & 2,39419 & 1,53733 \\
\hline
\end{tabular}

Fonte: Dados provenientes da coleta de dados.

Nota-se que o resultado da positivo da satisfação se deve principalmente aos itens relacionados a mercadoria e ao atendimento ofertado pelos comerciantes do Mercadão. Esses resultados vão ao encontro dos destacados na literatura revisada.

\section{CONSIDERAÇÕES FINAIS}

O objetivo principal deste estudo foi avaliar a imagem de loja e a satisfação do consumidor do Mercado Municipal de Campo Grande-MS. Por meio da análise dos dados obtidos, observou-se que os principais atributos de imagem de loja que contribuem para a satisfação, foram: mercadoria, layout e atendimento, pois obtiveram melhores avaliações. Esses mesmos atributos apareceram como relevantes nos estudos de (Silva et al. 2020; Bloemer \& Ruyter 1998; Watanabe, Lima-Filho e Torres, 2013; Rojo, 1998; Campolina et al. 2007; Graciola 2015; Silva e Giraldi, 2012) em diferentes contextos.

Verificou-se que os atributos "percepção de densidade" e o "projeto arquitetônico" não foram relevantes, ou seja, suas pontuações foram abaixo da média, talvez pelo fato de os consumidores já conhecerem o formato de loja do Mercado Municipal posto que a maioria das cidades possui um Mercado Municipal. Segundo Santos \& Borges (2019), os fatores culturais promovem maior influência sobre as decisões de consumo, neste caso o consumidor pode visualizar o Mercadão como uma marca gerando orgulho e confiança para compra e consumo no local.

Diante dos resultados apresentados sugere-se aos gestores e comerciantes do Mercadão o contínuo investimento em mercadorias, agregando novos produtos ao leque de opções ofertado no local, principalmente nos produtos que remetam à cultura sul-mato-grossense, pois o Mercadão recebe um bom fluxo de turistas, com atenção especial a qualidade das mercadorias vendidas. Em relação ao layout, a percepção do espaço ocupado (crowding) sugere a realização de um estudo cuidadoso de alteração no layout. Além disso, sugerem-se treinamentos aos comerciantes e trabalhadores para ressaltar a importância do bom atendimento e o quanto esse atributo impacta na imagem do estabelecimento.

0 questionário utilizado nesta pesquisa, baseado no estudo de Graciola (2015), se mostrou eficiente na identificação dos atributos que influenciam positivamente e negativamente na imagem do Mercadão de Campo Grande-MS, fornecendo aos gestores informações capazes de auxiliar na tomada de decisões mais assertivas para satisfazer seus clientes. No decorrer do estudo verificou-se uma limitação com a aplicação do questionário via formulário eletrônico. Para futuros estudos sugere-se a aplicação do questionário in loco para identificar se há diferenças nos resultados da pesquisa entre os respondentes.

Também como sugestão para trabalhos futuros, propõe-se a utilização do mesmo questionário em outros mercados municipais e outros tipos de varejo para verificar o comportamento dos consumidores e realizar comparações com o presente estudo. Outros aspectos como o valor hedônico e o valor utilitário podem ser acrescentados com o intuito de decifrar com mais riqueza o comportamento do consumidor. 


\section{Referências}

Aguiar, E. C., \& Policarpo, M. C. (2018). Fenomenologia da Percepção: Uma abordagem para a investigação de experiência de consumo. Consumer Behavior Review, 2(2), 72- 83.

Associação Brasileira de Supermercado (2019). Superhiper: conteúdo que transforma. Disponível em: https://www.abras.com.br/economia-e-pesquisa/ranking-abras/os-numeros-do-setor/

American Marketing Association (2014). Dictionary. Disponível em: <https://www.ama.org/resources/Pages/Dictionary.aspx?dLetter=B>. Acesso em: 23 jul.2014.

Baker, J., Berry, L. L., \& Parasuraman, A. (1998). The Marketing Impact of Branch Facility Design. Journal of Retail Banking, 10(2), 33-42.

Baker, J., Levy, M., \& Grewal, D. (1992). An experimental approach to making retail store environmental decisions. Journal of Retailing, 68(4), 445-460.

Baltas, G., \& Papastathopoulou, P. (2003). Shopper characteristics, product and store choice criteria: a survey in the Greek grocery sector. International Journal of Retail \& Distribution Management, 31(10), 498-507.

Batista, K., \& Escobar, M. A. R. (2020). A importância do visual merchandising na percepção dos consumidores. Revista Gestão e Análise. Journal of Management Analysis, 9(1), 93-108.

Bloemer, J., \& Ruyter, K. (1998). On the relationship between store image, store satisfaction and store loyalty. European Journal of Marketing, 32(5/6), 499-513.

Brun, H. M., Rodrigues, W. O. P., Finocchio, C. P. S., \& de SOUSA, C. C. (2019). Análise da margem de comercialização do sistema agroindustrial do leite da cidade de Campo Grande - MS. Anais do III Encontro Internacional de Gestão, Desenvolvimento e Inovação (EIGEDIN), 3(1).

Campolina, A.S., Barroso, A.C., Carvalho, A., Xavier, G., Oliveira, J., \& Dourado, M. (2007). Decisões de compra dos clientes de supermercados de Anápolis. Revista de Economia da UEG, 3(1), 125-145.

Costa, D. \& Falcão, R. F. (2018). O valor percebido pelos usuários na troca de tempo: o caso Bliive. Consumer Behavior Review, 2(Special Edition), 19-32.

Din, R. (2000). New Retail. Conran Octopus: London.

Glendinning, M. (2004). The last icons. The Lighthouse Scottish Architectural and Design Series, Graven Images: Glasgow.

Gomez, I. M., McLaughlin, W. E. \& Wittink, R. D. (2004). Customer satisfaction and retail sales performance: an empirical investigation. Journal of Retailing, 8(4), 265-78.

Graciola, A. P. (2015). A influência da imagem de loja, do valor percebido e do conhecimento de marca na intenção de compra em diferentes formatos de varejo. 2015. Dissertação (Mestrado em Administração) - Universidade de Caxias do Sul, Caxias do Sul, 2015. Disponível em: https://repositorio.ucs.br/handle/11338/1134.

Grewal, D., \& Baker, J. (1994). Do retail environmental factors affect consumers' price Acceptability? An empirical examination. International Journal of Research in Marketing, 11(2), 107-115.

Hair, J. F. et al. (2010). Multivariate data analysis. 7. Ed, ed. Prentice Hall: Upper saddle River, 2010.

Harris, K., Baron, S., \& Parker, C. (2000). Understanding the consumer experience: it's 'good to talk'. Journal of Marketing Management, 16(1-3), 111-127.

$\mathrm{Hu}, \mathrm{H}$. (2011). Chinese consumer's store image formation and its impact on patronage behaviour. Academic of Marketing Studies Journal, 15(2), 83-102.

$\mathrm{Hu}, \mathrm{H} .$, \& Jasper, C. R. (2006). Social cues in the store environment and their impact on store image. International Journal of Retail \& Distribution management, 34(1), 25-48.

Hui, M. K., \& Bateson, J. E. (1991). Perceived control and the effects of crowding and consumer choice on the service experience. Journal of Consumer Research, 18(2), 174-184.

Iyengar, S. S., \& Lepper, M. R. (2000). When choice is demotivating, can one desire too much of a good thing? Journal of Personality and Social Psychology, 79(6), 995-1006.

Jinfeng, W., \& Zhilong, T. (2009). The impact of selected store image dimensions on retailer equity: evidence from 10 Chinese hypermarkets. Journal of Retailing and Consumer Services, 16(6), 486494.

Jencks, C. (2005). The iconic building: the power of enigma. Frances Lincoln: London. 
Julier, G. (2005). Urban designscapes and the production of aesthetic consent. Urban Studies, 42(5), 869-887.

Kim, J.E., \& Kim, J. (2012). Human factors in retail environments: a review. International Journal of Retail \& Distribution Management, 40(11), 818-841.

Lima Filho, D. O. (1999). Valor percebido e o comportamento do consumidor de supermercado. 1999. Tese (Doutorado em Administração) - Escola de Administração de Empresas de São Paulo, Fundação Getúlio Vargas, São Paulo, 1999. Disponível em: https://bibliotecadigital.fgv.br/dspace/handle/10438/4519

Lindquist, J. D. (1974-75). Meaning of image: survey of empirical and hypothetical evidence. Journal of Retailing, 50(4), 29-38.

MacIntosh, G., \& Lockshin, L. (1997). Retail relationships and store loyalty: a multi-level perspective. International Journal of Research in Marketing, 14(5), 487-97.

Malhotra, N. K., Birks, D., \& Wills, P. (2012). Marketing research: apllied approach. 4. Ed. New York: Pearson.

Martineau, P. (1958). The personality of the retail store. Harvard Business Review, 36(1), 47-55.

Mittal, A., \& Mittal, R. (2008). Store choice in the emerging indian apparel retail market: na empirical analysis. Journal of Retailing, 77(2), 21-46.

Mohan, G., Sivakumaran, B., \& Sharma, P. (2013). Impact of store environment on impulse buying behavior. European Journal of Marketing, 47(10), 1711-1732.

Morgan, T. (2011). Visual merchandising: vitrines e interiores comerciais. 2. Ed. Barcelona: Editora GG, 2011.

Morrison, G. P. (2006). Driving successful shopping occasions through deeper insights. European Retail Digest, 49, 65-75.

Oliver, R. L. (1980). A cognitive model of the antecedents and consequences of satisfaction decisions. Journal of Marketing Research, 17(4), 460-469.

Oliver, R. L. (1981). Measurement and evaluation of satisfaction processes in retail settings. Journal of Retailing, 57, 25-48.

Parente, J. (2007). Varejo no Brasil. São Paulo: Atlas, 2007.

Queiroz, T. (2019). Berço da cultura, culinária e ponto turístico, Mercadão é amado por MS. Jornal Correio do Estado. Disponível em: https://www.correiodoestado.com.br/cidades/berco-dacultura-culinaria-e-ponto-turistico-mercadao-e-amado-por-ms/344997/. Acesso em 11 jul. 2019.

Rojo, F. J. G. (1998). Pesquisa: 0 comportamento do consumidor nos supermercados. Revista de Administração de Empresas, 38(3), 16-24.

Santos, L. L., \& Borges, G. R. (2019). Fatores que influenciam no consumo de carne ovina. Consumer Behavior Review, 3(1), 42-56.

Silva, K. A., Teixeira, É., \& Soares, U. G. (2020). A influência do layout como fator de crescimento nas vendas no varejo: um estudo de caso na empresa Realce Moda e acessórios em João Pinheiro MG. Scientia Generalis, 1(1), 22-37.

Silva, T. S., \& Giraldi, J. M. E. (2012). Um estudo empírico sobre as dimensões da imagem de loja no varejo calçadista. Revista de Gestão da USP, 19(1), 125-143.

Soars, B. (2009). Driving sales through shoppers' sense of sound, sight, smell and touch. International Journal of Retail \& Distribution Management, 37(3), 286-298.

Stern, B., Zinkhan, G. M., \& Jaju, A. (2001). Marketing Images: construct definition, measurement issues, and theory development. Marketing Theory, 1(2), 201-224.

Swani, K., \& Yoo, B. (2010). Interactions between price and price deal. Journal of Product \& Brand Management, 19(2), 143-152.

Theodoridis, P. K., \& Chatzipanagiotou, K. C. (2009). Store Image attributes and customer satisfaction across different customer profiles within the supermarket sector in Greece. European Journal of Marketing, 43(5/6), 708-734.

Tonetto, L. M., Brust-Renck, P. G., \& Stein, L. M. (2014). Perspectivas metodológicas na pesquisa sobre o comportamento do consumidor. Psicologia: ciência e profissão, 34(1), 180-195. 
Van Der Vyver, J. (2008). The importance of store image dimensions in apparel. 2008. 175p. Dissertação de Mestrado, Universidade de Stellenbosch, 2008.

Wang, L. C., Chang, L. H., \& Wysong, S. (2012). An empirical investigation of the influence of optimum stimulation levels in retailing. International Journal of Retail \& Distribution Management, 40(1), 6-20.

Watanabe, E. A. DE M. (2014). A influência da cultura no comportamento do consumidor de supermercados. Brasília: UNB, 2014. Tese (Doutorado em Administração), Faculdade de Economia, Administração e Contabilidade, Universidade de Brasília, 2014. Disponível em: https://repositorio.unb.br/handle/10482/16075.

Watanabe, E.A.M., Lima-filho, D.O., \& Torres, C.V. (2013). Store image attributes and customer satisfaction in supermarkets in Campo Grande-MS. Revista Brasileira de Marketing, 12(4), 85107. 